\title{
Globe
}

Revue internationale d'études québécoises

\section{Annette Hayward : La querelle du régionalisme au Québec (1904-1931). Vers l'autonomisation de la littérature québécoise, Ottawa, Le Nordir, 2006}

\section{Michel Lacroix}

Volume 10, numéro 1, 2007

URI : https://id.erudit.org/iderudit/1000121ar

DOI : https://doi.org/10.7202/1000121ar

Aller au sommaire du numéro

Éditeur(s)

Globe, Revue internationale d'études québécoises

ISSN

1481-5869 (imprimé)

1923-8231 (numérique)

Découvrir la revue

Citer ce compte rendu

Lacroix, M. (2007). Compte rendu de [Annette Hayward : La querelle du régionalisme au Québec (1904-1931). Vers l'autonomisation de la littérature québécoise, Ottawa, Le Nordir, 2006]. Globe, 10(1), 188-190.

https://doi.org/10.7202/1000121ar d'utilisation que vous pouvez consulter en ligne.

https://apropos.erudit.org/fr/usagers/politique-dutilisation/ 
problématique plus serrée, l'ouvrage ne réussit pas non plus à développer des thèmes plus larges (l'exceptionnalisme québécois, l'impact de la mondialisation ou du néolibéralisme sur les politiques publiques), qui auraient pu alimenter des débats transdisciplinaires sur le Québec.

Bref, malgré son intention de mettre le Québec en contexte, le collectif ne réussit pas à fournir un cadre théorique unifié qui justifierait la comparaison. Le choix de la comparaison en est un surtout méthodologique, motivé par sa capacité à approfondir une problématique ou une question de recherche précise. Le résultat, dans le cas de ce livre, est une série de chapitres aux problématiques fort différentes, qui avancent très peu notre appréciation de la place du Québec dans ses contextes géographiques (continental, mondial) ou temporels (modernité, postmodernité, capitalisme post-industriel, etc.).

Peter Graefe

Université de McMaster

\section{Annette Hayward \\ La querelle du régionalisme au Québec (1904-1931). Vers l'autonomisation de la littérature québécoise Ottawa, Le Nordir, 2006.}

Cette publication recèle un paradoxe, propre à désarçonner la critique. En 1980, Annette Hayward soutenait une thèse de plus de 1000 pages, intitulée "Le conflit entre les régionalistes et les exoriques au Québec (1900-1920)"; depuis, bien peu de publications consacrées au régionalisme, au Nigog ou aux premières décennies du XX $\mathrm{XX}^{e}$ siècle ne s'y réfèrent pas. Désormais, les chercheurs devront refaire leurs notes, pour référer au livre. Ils n'auront cependant pas à le relire, puisqu'à l'exception de très légers retranchements et de retouches stylistiques mineures, rien n'a été changé au texte initial.

Cette parution d'un texte dont la fortune critique est un fait avéré, lui-même susceptible d'une étude historique, constitue à toutes fins pratiques une réédition. Il nous parvient d'ailleurs pourvu d'un discours d'escorte caractéristique: une préface de Dominique Garand et une brochure publicitaire rassemblant les éloges de Marie-Andrée Beaudet, Jacques Michon et François Ricard. Bref, voilà un classique de la critique littéraire historique, désormais plus accessible. 
À la suite de ce quatuor d'intercesseurs, nous ne pouvons que célébrer l'ampleur et la minutie de l'érudition d'Annette Hayward (pas moins de 2000 notes accompagnent le texte), laquelle a dépouillé des dizaines de journaux, revues et monographies pour reconstituer, pas à pas, l'élaboration puis la confrontation des deux grandes écoles esthétiques de la période, celles des régionalistes et des "exotiques". La plus grande force, sans doute, de l'ouvrage d'Hayward est d'ailleurs imputable à cette abondante documentation, dans la mesure où elle permet d'appréhender historiquement la querelle, d'introduire du mouvement là où on n'a trop souvent vu que la domination d'un monolithique et immuable régionalisme, de Gérin-Lajoie à Ringuet. L'attention portée à la complexité des positions, à l'évolution des discours, à l'apport respectif des divers acteurs fait qu'on retourne régulièrement à ce texte, comme à un vade-mecum.

Presque rien, du texte original, n’a été modifié, ai-je dit. Et pourtant, deux modifications sont révélatrices. La première concerne l'introduction, qui comportait originellement une section intitulée "état des connaissances", laquelle a disparu de la présente édition. On comprend aisément pourquoi ces pages disparurent: cet "état présent" est désormais désuet, contrairement au reste de l'ouvrage, et en grande partie à cause du renouveau dans l'étude du régionalisme initié par cet ouvrage ainsi que de la "résurrection" du Nigog qu'on lui doit. De plus, les rares travaux signalés en 1980, par Hayward, ne jouaient aucun rôle dans l'analyse. Cette coupure s'imposait donc et pouvait s'effectuer sans modifier le reste du texte. On peut cependant se demander pourquoi l'auteure n'a pas jugé pertinent de faire le point, dans une postface par exemple, sur l'état actuel des travaux dans le domaine. Depuis la thèse, une kyrielle de contributions majeures ont approfondi notre conception du régionalisme, au Québec comme en France: pensons entre autres aux ouvrages de Marie-Andrée Beaudet (1991), Dominique Garand (1989) et Anne-Marie Thiesse (1991), à la cinquième livraison de La vie littéraire au Québec (2005), qui couvre les années 18951918, ou encore aux textes rassemblés dans le tout récent collectif L'artiste et ses lieux. Les régionalismes de l'entre-deux-guerres face à la modernité. De même, la relecture de l'histoire intellectuelle et politique de la période a modifié en profondeur la vision du nationalisme canadien-français et a remis en lumière le rôle des courants de pensée associés au libéralisme (mentionnons par exemple les travaux d'Yvan Lamonde, de Fernande Roy, de Pierre Trépanier, pour ne nommer qu'eux). Or, à l'exception du livre de Garand (qui préface l'ouvrage), et d'articles ultérieurs d'Annette Hayward 
elle-même, cités dans la bibliographie, La querelle du régionalisme au Québec les passe sous silence. Une occasion a été manquée, ici, d'opérer un retour sur le texte de 1980. Tout se passe ainsi comme si la version de 2006 n'était pas, n'était plus datée, comme si une période de l'histoire littéraire du Québec était saisie en elle-même, par un observateur hors du temps, pour qui les notions utilisées n'appartiennent pas à l'histoire de la critique mais à celle du contexte étudié.

Et pourtant, on peut juger que ces notions ne vont pas de soi. Ainsi de celle d'autonomisation, qui est venue éclairer le sous-titre mais n'y était pas dans la version initiale. Avec ce changement, mineur en apparence, puisqu'il n'affecte pas le contenu du texte, Hayward s'inscrit dans une filiation très forte, celle de la sociologie de la littérature issue de Pierre Bourdieu. Certes, la thèse formulée dans le dernier chapitre de La querelle $d u$ régionalisme, à savoir qu'avec la diversification idéologique des années vingt et l'affaiblissement du régionalisme traditionnel, la littérature québécoise entre dans la voie de l'autonomie, correspond dans ses grandes lignes à l'hypothèse bourdieusienne de l'établissement d'un champ littéraire autonome, bien que le sens accordé au terme demeure plutôt métaphorique. De même, on ne peut guère reprocher au texte de Hayward de n'évoquer qu'en passant les travaux de Bourdieu, puisque ses apports majeurs à l'histoire du champ littéraire sont postérieurs à 1980. Toutefois, le changement est significatif. Convoquer en sous-titre une notion relativement secondaire et plutôt librement utilisée, dans le texte, bien qu'elle soit au cœur des travaux depuis vingt ans et s'inscrive dans un cadre théorique précis et complexe, témoigne de l'importance secondaire accordée à la théorie. Là réside, en partie, bien que paradoxalement, la force du texte: en s'effaçant derrière la reconstitution des discours des acteurs, sans recourir aux grilles théoriques qui pullulaient pourtant dans les années 1970 et 1980 , il opère une patiente et "transparente" archéologie, pour reprendre le terme de François Ricard. Cette force peut cependant s'avérer, sous un autre angle, une faiblesse notable; autonomie, idéologie, modernité, régionalisme, etc., ces termes ne sont pratiquement jamais définis, par Hayward, bien qu'ils soient au cour de ses analyses.

Michel Lacroix Université du Québec à Trois-Rivières 